\title{
Discrepant responses of the global electron content to the solar cycle and solar rotation variations of EUV irradiance
}

\author{
Yiding Chen ${ }^{1,2^{*}}$, Libo Liu' ${ }^{1,2}$, Huijun Le ${ }^{1,2}$ and Hui Zhang ${ }^{1,2}$
}

\begin{abstract}
In this paper, the responses of the ionosphere to the solar cycle and solar rotation variations of extreme ultraviolet (EUV) irradiance are comparatively investigated using daily mean global electron content (GEC) and 0.1-50 nm EUV daily flux. GEC is well correlated with EUV on both the solar cycle and solar rotation timescales; however, the responses of GEC to the solar cycle and solar rotation variations of EUV are significantly different in terms of the following two aspects: (1) There is a significant time lag between the solar rotation variations of GEC and EUV; the lag is dominated by a 1-day lag and generally presents a decrease trend with solar activity decreasing. For the solar cycle variations of GEC and EUV, however, there are no evident time lags. (2) The GEC versus EUV slopes are different for the solar cycle and solar rotation variations of GEC and EUV; the solar cycle GEC versus EUV slope is higher than the solar rotation GEC versus EUV slope, and this difference occurs in different seasons and latitudinal bands. The results present an aspect of the difference between ionospheric climatology and weather.
\end{abstract}

Keywords: Ionosphere; Solar cycle variation; Solar rotation variation

\section{Findings}

\section{Background}

The solar extreme ultraviolet (EUV) irradiance results in the photoionization of atmospheric neutral particles to generate ionospheric plasma; thus, its variations play important roles in ionospheric variability. The solar EUV varies on various timescales (e.g., Bouwer 1992; Chen et al. 2012; Lean et al. 2011; Pap et al. 1990; Tobiska and Bouwer 1989); the most prominent variations of EUV include the $\sim 11$-year quasi-periodic variation (the solar cycle variation) and the $\sim 27$-day quasi-periodic variation (the solar rotation variation). The solar cycle variation is the result of the reversal of the solar magnetic polarity (Lean 1997), while the solar rotation variation is induced by the rotation of the Sun and the evolution of solar active regions (Bouwer 1992). The solar rotation variation of EUV changes during solar cycles (e.g., Kane 2003), stronger at solar maxima and weaker at solar minima,

\footnotetext{
* Correspondence: chenyd@mail.iggcas.ac.cn

'Key Laboratory of Earth and Planetary Physics, Institute of Geology and Geophysics, Chinese Academy of Sciences, Beijing 100029, China

${ }^{2}$ Beijing National Observatory of Space Environment, Institute of Geology and Geophysics, Chinese Academy of Sciences, Beijing 100029, China
}

since active regions are closely related to solar activity levels (e.g., Lean 1987). Both the solar cycle and solar rotation variations of EUV significantly affect the ionosphere; The former causes a significant solar cycle modulation in the ionosphere (e.g., Bilitza 2000; Chen et al. 2011; Liu et al. 2011), and the latter modulates ionospheric variations on the timescales of days (e.g., Chen et al. 2014a; Coley and Heelis 2012; Forbes et al. 2000; Hocke 2008; Min et al. 2009; Oinats et al. 2008; Rich et al. 2003; Wang et al. 2006).

The responses of the ionosphere to EUV variations have been investigated in many studies (e.g., Balan et al. 1994; Chen and Liu 2010; Hocke 2008; Liu et al. 2006; Richards 2001; Sethi et al. 2002); however, the quantitative relationships between ionospheric variations and the long-term (solar cycle timescales) and short-term (solar rotation timescales) variations of EUV were barely comparatively investigated. Rishbeth (1993) investigated the responses of the ionosphere to solar irradiance variations during a high solar activity period using the ionosonde data over Slough, Port Stanley, and Huancayo. He found that the relationship between short-term variations of the E-layer electron density and the solar irradiance is 
basically consistent with the relationship between their longer-term variations; for the F-layer, however, he found that short-term variations of the F-layer electron density and EUV irradiance lack strong correlations. In this paper, we pay attention to the consistency of the responses of the F-layer to the solar cycle and solar rotation variations of EUV.

As far as ionospheric response to the solar rotation variation of EUV is concerned, some studies revealed that ionospheric variations are usually most correlated with the EUV variations of previous days or hours, i.e., there are time lags (e.g., Coley and Heelis 2012; Min et al. 2009; Rich et al. 2003; Wang et al. 2006). The thermosphere is the background of the ionosphere; its variations significantly affect ionospheric variability. The time lags were found to also exist in thermospheric responses to the changes of the solar irradiance (e.g., Buonsanto and Pohlman 1998; Eastes et al. 2004; Jakowski et al. 1991). Some studies suggested that the lags of the ionosphere to the solar irradiance are possibly related to the lags of the thermosphere to the solar irradiance (e.g., Min et al. 2009; Wang et al. 2006). The solar cycle variation of solar irradiance also significantly modulates the thermosphere (e.g., Emmert et al. 2010; Liu et al. 2005; Solomon et al. 2010, 2011), which should affect the solar cycle variation of the ionosphere. Thus, it is essential to investigate whether the response of the ionosphere to the solar cycle variation of EUV has evident time lags.

This research comparatively investigated the responses of the ionosphere to the solar cycle and solar rotation variations of EUV using daily mean global electron content (GEC) and the daily $0.1-50 \mathrm{~nm}$ EUV integral flux observed by the Solar EUV Monitor (SEM) aboard the Solar Heliospheric Observatory (SOHO) satellite (Judge et al. 1998). We focus on two features of GEC responses to EUV, time lags and quantitative relationships of GEC versus EUV. The parameter GEC was used since its short-term variation is well correlated to EUV shortterm variation, thus, the time lags and the quantitative relationship between the short-term variations of the ionosphere and EUV can be reliably derived. The results of this paper indicate that ionospheric responses to the solar cycle and solar rotation variations of EUV are significantly different.

\section{Data}

GEC was suggested to be a good indicator for presenting solar EUV effects on the ionosphere (Afraimovich et al. 2008; Chen et al. 2012; Hocke 2008). In this paper, daily mean GECs were calculated from the total electron content (TEC) maps of the Jet Propulsion Laboratory (JPL) to investigate ionospheric responses to the solar cycle and solar rotation variations of EUV. The JPL TEC maps have been routinely produced from the measurements of global GPS receivers since late 1998 (Iijima et al. 1999; Mannucci et al. 1998). The TEC maps are presented with a grid of $5^{\circ}$ in longitude by $2.5^{\circ}$ in latitude. There are 12 maps within each UT day. GECs were derived one by one from the JPL TEC maps by calculating the area integral of TEC in terms of the map grid according to Afraimovich et al. (2008). GEC is measured with the unit of GECu ( $1 \mathrm{GECu}=10^{32}$ electrons). For each UT day, a daily mean GEC was obtained by averaging the 12 GECs derived from the 12 TEC maps. The daily mean GECs of geomagnetically disturbed days $(A p>30$ in the previous or current day of GEC) were not analyzed to depress geomagnetic activity effects, since the purpose of this paper is to investigate solar EUV effects.

The actual Sun-Earth distance has a $\sim 3.5 \%$ variation during a year, which causes a $\sim 7 \%$ variation of the solar irradiance that arrives at the Earth. The downloaded SOHO/SEM daily EUV flux is the dataset normalized to 1 AU. Thus, we revised the SOHO/SEM 0.1-50 nm EUV flux according to the actual Sun-Earth distance in order to more accurately obtain the quantitative relationship between GEC and EUV. It should be noted that EUV observations aboard satellites usually have instrument degradations. This degradation also exists in the SOHO/SEM recent measurements (Didkovsky and Wieman, 2014; Solomon et al., 2013; Wieman et al., 2014), which possibly affects the results of EUV long-term variations to some extent. This effect is not prominent for the SOHO/SEM EUV flux measurements in solar cycle 23 according to the calibrations with other EUV measurements shown in Didkovsky et al. (2010) and Solomon et al. (2010). Therefore, only the GEC and EUV data before the end of 2007 were used in this analysis.

On longer-term timescales, EUV variations mainly include the solar cycle variation, while ionospheric variations also include seasonal variations in addition to the solar cycle variation. A 365-day (1-year window) running average can be used to remove seasonal variations of GEC as well as the shorter-term variations of GEC and EUV (including the solar rotation variation). In this paper, we use 365-day running averaged GEC and EUV (denoted by $\mathrm{GEC}_{365 \mathrm{~A}}$ and $\mathrm{EUV}_{365 \mathrm{~A}}$ ) to estimate the solar cycle variations of GEC and EUV. Figure 1a shows the variations of 365-day running averaged GEC and EUV during solar cycle 23. GEC and EUV display very consistent solar cycle variations. The Lomb-Scargle periodograms of 365-day running averaged GEC and EUV are shown in Fig. 1c to further verify this consistency. A 27-day running average can be used to separate the variations of GEC and EUV on the timescales of days from the datasets (e.g., Chen et al. 2014a). We obtained 27-day average residual GEC and EUV via subtracting 27-day running averaged GEC and EUV from the 


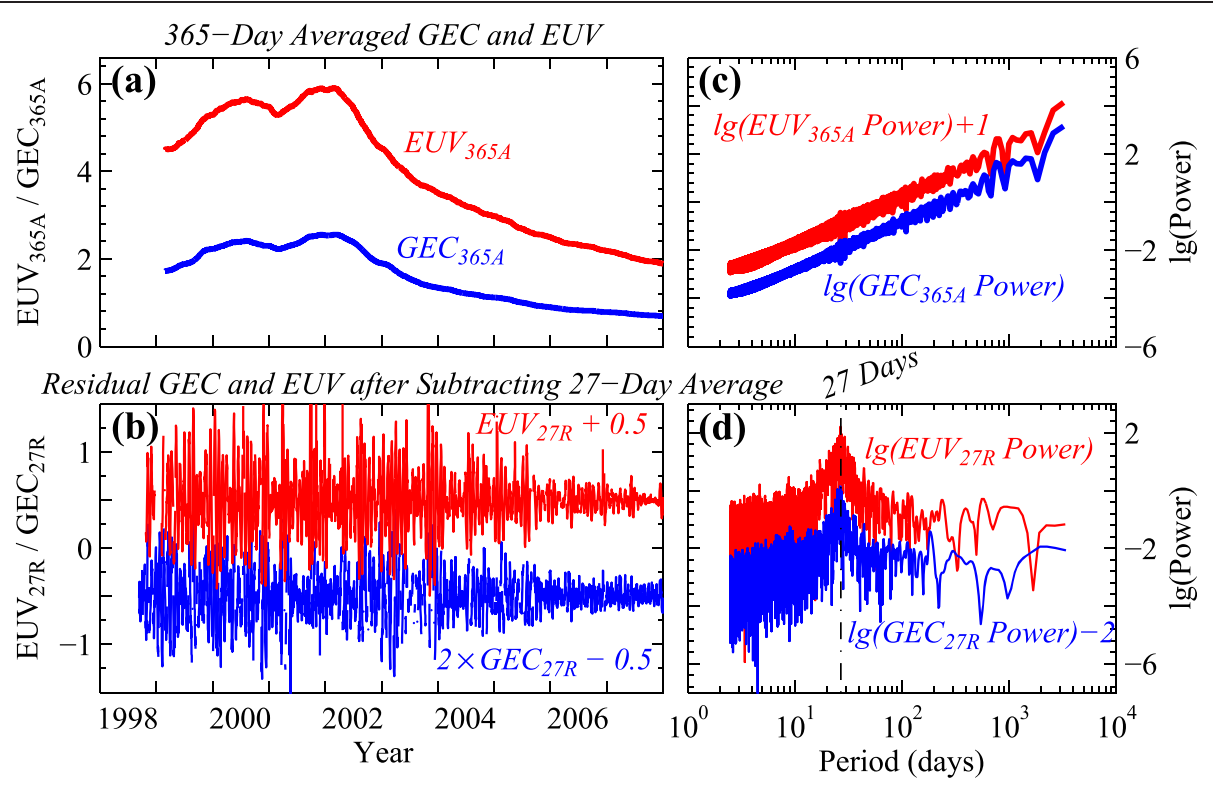

Fig. 1 Time series (left) and Lomb-Scargle periodograms (right) of GEC and EUV. $\mathbf{a}$ and $\mathbf{c}$ are the results for 365-day running averaged GEC and EUV, and $\mathbf{b}$ and $\mathbf{d}$ are the results for 27-day average residual GEC and EUV. GEC and EUV are in units of $10^{32}$ electrons and $10^{10} \mathrm{photons} / \mathrm{cm}^{2} / \mathrm{s}$, respectively

datasets. The 27-day average residual GEC and EUV (denoted by $\mathrm{GEC}_{27 \mathrm{R}}$ and $\mathrm{EUV}_{27 \mathrm{R}}$ ) indicate the variations of GEC and EUV on the timescales of days; they are used to estimate the solar rotation variations of GEC and EUV. Figure 1b shows 27-day average residual GEC and EUV; both are very variable and generally larger at higher solar activity levels than at lower solar activity levels. The Lomb-Scargle periodograms of $\mathrm{GEC}_{27 \mathrm{R}}$ and $E V_{27 R}$ shown in Fig. 1d indicate that both $G C_{27 R}$ and $\mathrm{EUV}_{27 \mathrm{R}}$ are dominated by the solar rotation variation. The power spectra of $\mathrm{GEC}_{27 \mathrm{R}}$ and $\mathrm{EUV}_{27 \mathrm{R}}$ are similar, implying that EUV irradiance is the primary reason for GEC variations on shorter-term timescales.

\section{Results and discussion \\ Time lags of GEC to EUV}

Time lags of the responses of GEC to the solar cycle and solar rotation variations of EUV were investigated based on 365-day running averaged GEC and EUV and 27-day average residual GEC and EUV. Cross correlation analyses were used to estimate time lags of GEC to EUV. The time lag corresponds to the lag value of GEC to EUV for which the correlation coefficient between GEC and EUV reaches a maximum. Figure 2 presents an example to illustrate how a time lag of GEC to EUV is derived using $G C_{27 R}$ and $E V_{27 R}$. The correlation coefficient between $\mathrm{GEC}_{27 \mathrm{R}}$ and $\mathrm{EUV}_{27 \mathrm{R}}$ changes when different lag values are used; it peaks when the lag value of 2 days is used. Thus, the time lag of $\mathrm{GEC}_{27 \mathrm{R}}$ to $E V_{27 R}$ equals to 2 days for this data bin.
For the solar rotation variations of GEC and EUV, time lags were derived via cross correlation analyses for the $\mathrm{GEC}_{27 \mathrm{R}}$ and $\mathrm{EUV}_{27 \mathrm{R}}$ within 81-day running bins with a steplength of 1 day. Figure 3a shows the evolution of the correlation coefficient between $\mathrm{GEC}_{27 \mathrm{R}}$ and $\mathrm{EUV}_{27 \mathrm{R}}$; the results are not used if the correlation coefficients are less than 0.7 in order to ensure high statistical significance. $\mathrm{GEC}_{27 \mathrm{R}}$ and $\mathrm{EUV}_{27 \mathrm{R}}$ are well correlated over most temporal segments, implying the solar EUV is the primary reason for GEC variations on short-term timescales. There are three possible time lags (0-day, 1-day, and 2-day lags), with EUV leading GEC. The percentage

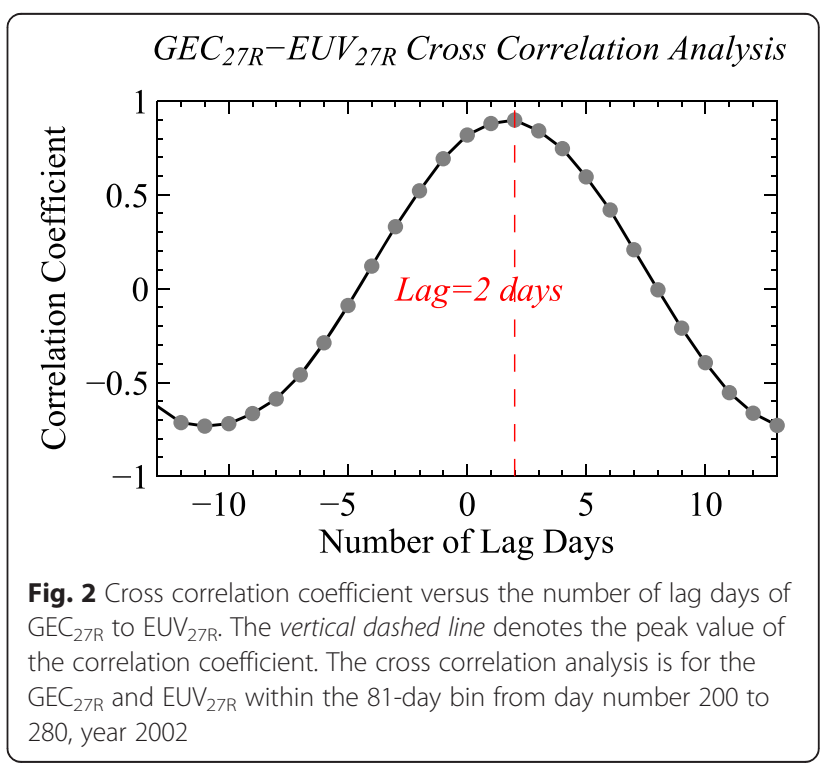



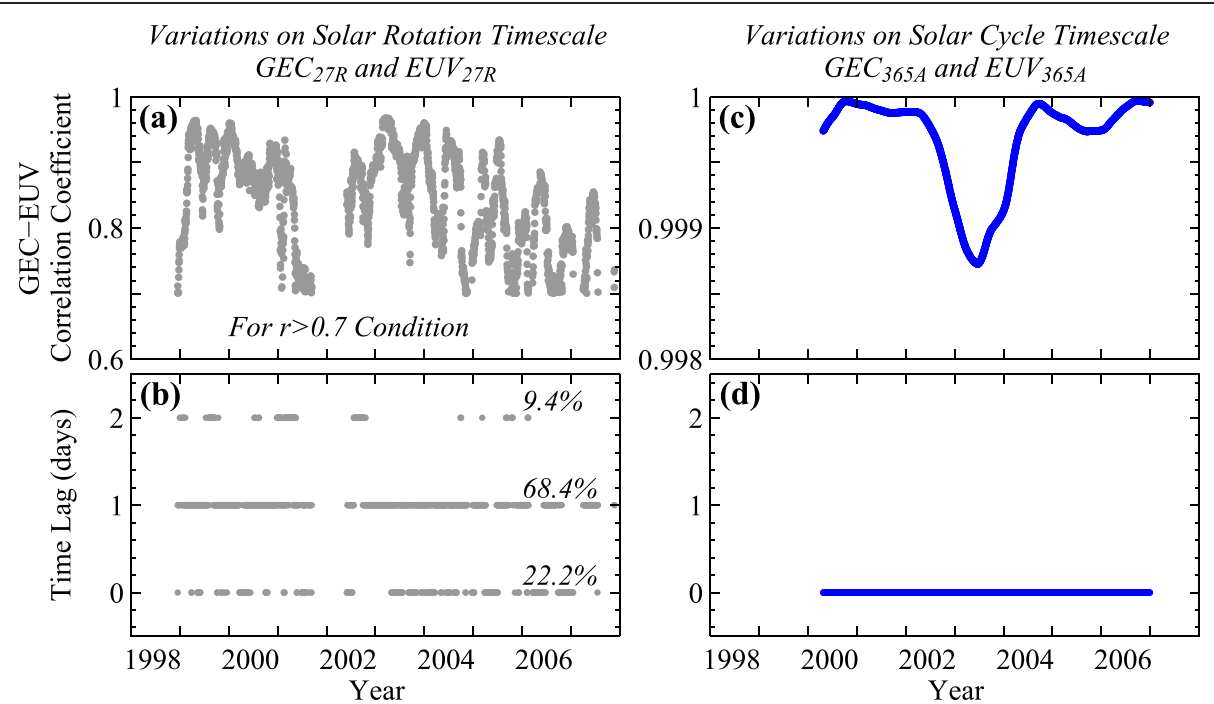

Fig. 3 Correlation coefficients between GEC and EUV (top) and time lags of GEC to EUV (bottom). $\mathbf{a}$ and $\mathbf{b}$ are the results for 27-day average residual GEC and EUV. The correlation coefficients and time lags are the statistical values for the GEC $_{27 R}$ and EUV $27 \mathrm{R}$ within 81 -day running bins with a 1-day steplength. The percent in $\mathbf{b}$ are percentage compositions of the three lags. $\mathbf{c}$ and $\mathbf{d}$ are similar to $\mathbf{a}$ and $\mathbf{b}$ but for 365-day running averaged GEC and EUV are used and the statistical values are for the $\mathrm{GEC}_{365 \mathrm{~A}}$ and $\mathrm{EUV}_{365 \mathrm{~A}}$ within 2-year running bins with a 1-day steplength

compositions of the three lags are $22.2 \%, 68.4 \%$, and $9.4 \%$, respectively, i.e., the 1 -day lag is dominant. For the solar cycle variations of GEC and EUV, time lags were derived via cross correlation analyses for the $\mathrm{GEC}_{365 \mathrm{~A}}$ and $\mathrm{EUV}_{365 \mathrm{~A}}$ within 2-year running bins with a steplength of 1 day. The 2-year bins were used since generally solar activity has evident changes over that timescale except at solar minima (see Fig. 1a). Figure 3c indicates that $\mathrm{GEC}_{365 \mathrm{~A}}$ and $\mathrm{EUV}_{365 \mathrm{~A}}$ within 2-year running bins are highly correlated. As for time lags, however, the case is significantly different from that of $\mathrm{GEC}_{27 \mathrm{R}}$ and $\mathrm{EUV}_{27 \mathrm{R}}$. Figure $3 \mathrm{~d}$ shows that no evident time lags occur for $\mathrm{GEC}_{365 \mathrm{~A}}$ and $\mathrm{EUV}_{365 \mathrm{~A}}$. In fact, there are no evident lags of GEC to EUV even if original datasets of daily mean GEC and EUV (including the solar cycle and solar rotation variations of GEC and EUV and being dominated by the solar cycle variations) are used without extracting the solar cycle variations of GEC and EUV from the datasets via 365-day running averages.

For the solar rotation variations of GEC and EUV, the dependence of the time lag on solar activity levels was investigated by analyzing the percentage compositions of the three time lags (0-day, 1-day, and 2-day) in different years. Figure 4 shows that the time lag presents a decrease trend with solar activity decreasing. In general, the composition of the 2-day lag is higher at higher solar activity levels (except for the year 2000) than at lower solar activity levels, and the composition of the 0-day lag tends to be higher at lower solar activity levels (except for the year 2007) than at higher solar activity levels.

The most direct influence of solar EUV variations on the ionosphere is to cause the change of photoionization. This influence does not directly result in the time lag of GEC to EUV. Ionospheric electron density depends on not only the photoionization but also the background thermosphere and ionospheric dynamics and electrodynamics processes. Thus, some studies attributed ionospheric lags to the solar irradiance to thermospheric lags to the solar irradiance (e.g., Min et al. 2009; Wang et al. 2006). Although the solar cycle variation of the solar irradiance also modulates the background thermosphere, there are no evident time lags between the solar cycle variations of GEC and EUV. A difference between the solar cycle and solar rotation variations of EUV is that the former is slower than the latter. Figure 5 shows the evolution of the difference between the current

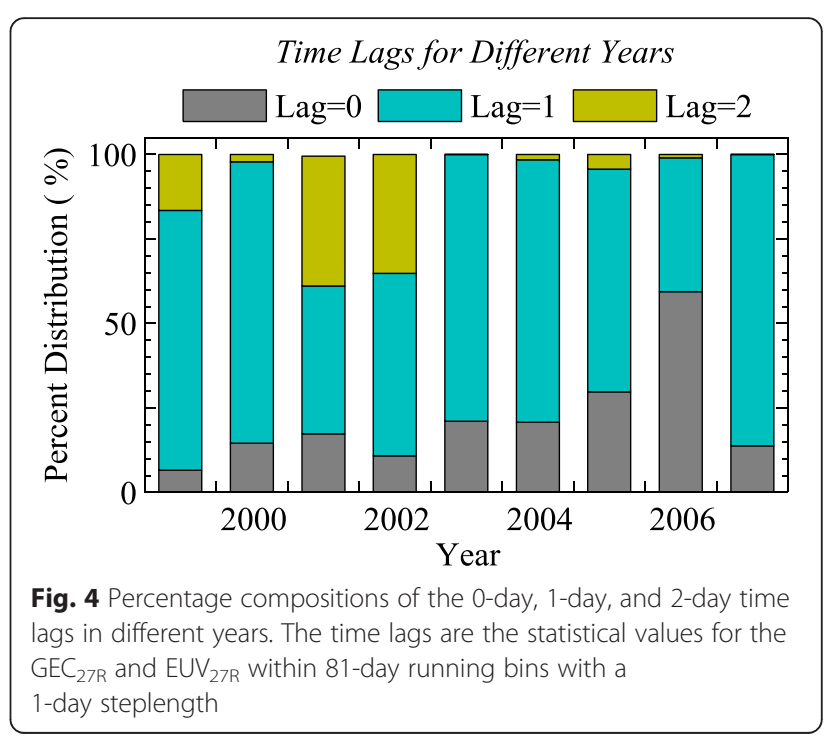


day EUV and the previous day EUV (the variation rate of EUV) during the entire period 1999-2007. The variation rate of EUV's solar rotation variation is generally significantly higher than that of EUV's solar cycle variation. The averages of the absolute value of $\triangle \mathrm{EUV}$ equal to 0.78 and 0.02 (in units of $10^{9}$ photons $/ \mathrm{cm}^{2} / \mathrm{s}$ ) for the solar rotation variation (Fig. 5a) and the solar cycle variation (Fig. 5b), respectively. Figure $5 \mathrm{a}$ indicates that the variation rate of EUV's solar rotation variation depends on solar activity levels, larger at higher solar activity levels than at lower solar activity levels. Meanwhile, for the solar rotation variations of GEC and EUV, the lag of GEC to EUV presents a decrease trend with solar activity decreasing. If the variation rate of EUV is an important determinant for the lag value of GEC to EUV, then the difference of the lag between the solar cycle and solar rotation variations of GEC and EUV may be understandable to some extent, because the variation rate of EUV's solar cycle variation is much lower than that of EUV's solar rotation variation. However, that cannot be certified in the current analysis.

\section{Variation slopes of GEC versus EUV}

Variation slopes of GEC versus EUV were investigated respectively for the solar cycle and solar rotation variations of GEC and EUV. Figure $6 \mathrm{a}, \mathrm{b}$ shows the scatter plots of $\mathrm{GEC}_{365 \mathrm{~A}}$ versus $\mathrm{EUV}_{365 \mathrm{~A}}$ and $\mathrm{GEC}_{27 \mathrm{R}}$ versus $E V_{27 R}$, respectively. In view of the dominative 1-day lag of $\mathrm{GEC}_{27 \mathrm{R}}$ to $\mathrm{EUV}_{27 \mathrm{R}}$, the $\mathrm{EUV}_{27 \mathrm{R}}$ used in Fig. $6 \mathrm{~b}$ is the value in the previous day of GEC. $G C_{27 R}$ and

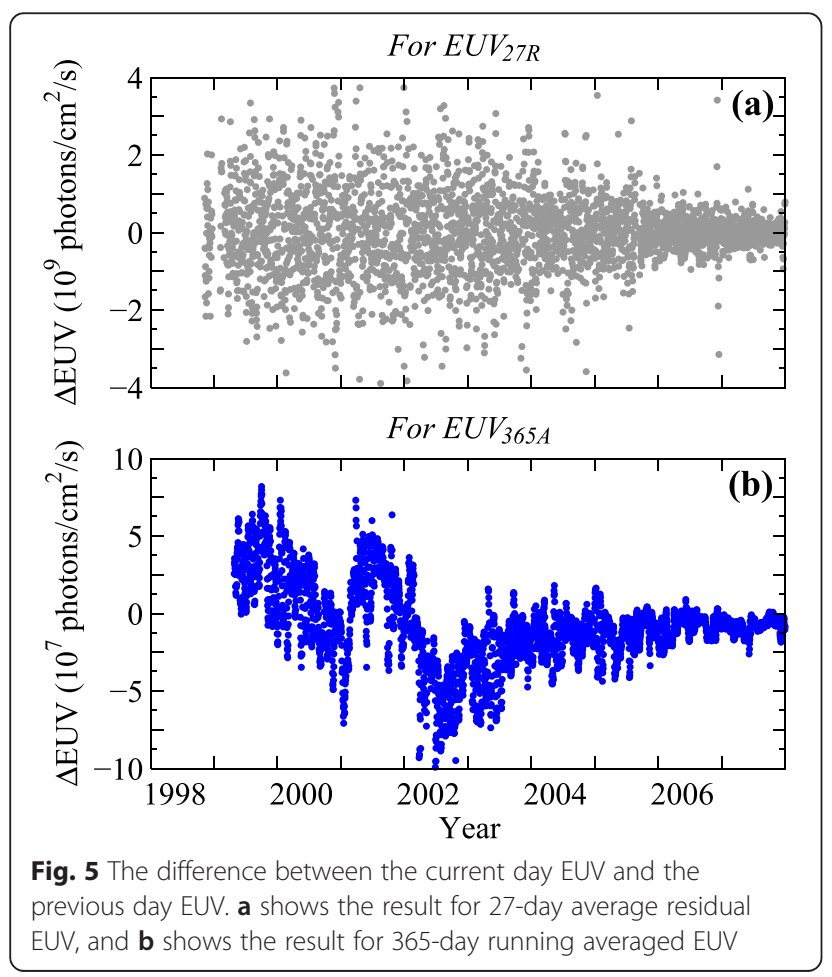

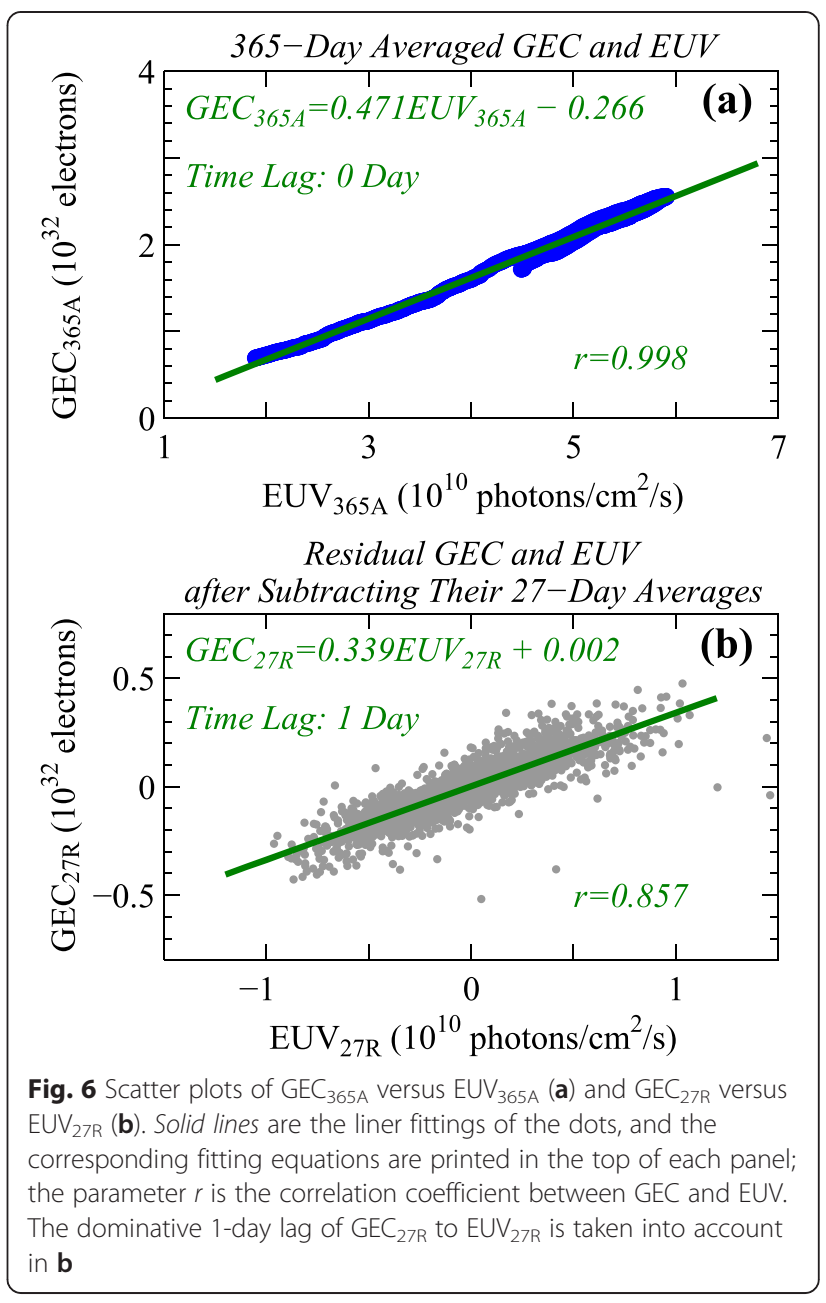

$E V_{27 R}$ are well correlated, different from the poorer correlation between the variations of the peak electron density and the solar irradiance on shorter-term timescales (e.g., Rishbeth 1993). Linear fittings were used to estimate variation slopes of GEC versus EUV. The variation slope of $\mathrm{GEC}_{365 \mathrm{~A}}$ versus $\mathrm{EUV}_{365 \mathrm{~A}}$ is significantly higher than that of $\mathrm{GEC}_{27 \mathrm{R}}$ versus $\mathrm{EUV}_{27 \mathrm{R}}$, i.e., GEC response to the solar rotation variation of EUV is different from GEC response to the solar cycle variation of EUV.

As revealed by previous studies (e.g., Chen and Liu 2010; Chen et al. 2014b), the response of the ionosphere to the solar EUV is seasonally dependent. This is not taken into account in the above analysis. Therefore, it is necessary to investigate whether the difference between GEC responses to the solar rotation and solar cycle variations of EUV occurs in all seasons. As mentioned above, the solar rotation variations of GEC and EUV were obtained by subtracting 27-day running averaged GEC and EUV (denoted by $\mathrm{GEC}_{27 \mathrm{~A}}$ and $\mathrm{EUV}_{27 \mathrm{~A}}$, respectively). $\mathrm{GEC}_{27 \mathrm{~A}}$ mainly includes the solar cycle and also seasonal variations of GEC. In general, seasonal variations of ionospheric electron density are dominated by 
the annual and semiannual variations (e.g., Liu et al. 2009). Then, $\mathrm{GEC}_{27 \mathrm{~A}}$ can be approximately estimated with $\mathrm{EUV}_{27 \mathrm{~A}}$ and day number of year (DoY) according to the following equation if the solar cycle variation of EUV is assumed to linearly affect GEC.

$$
\begin{aligned}
\mathrm{GEC}_{27 A}= & \sum_{i=0}^{2}\left[\left(a_{i} \cdot \mathrm{EUV}_{27 A}+b_{i}\right) \cdot \cos \left(\frac{2 \pi i \cdot \mathrm{DoY}}{365}\right)\right] \\
& +\sum_{i=0}^{2}\left[\left(c_{i} \cdot \mathrm{EUV}_{27 A}+d_{i}\right) \cdot \sin \left(\frac{2 \pi i \cdot \mathrm{DoY}}{365}\right)\right] \\
= & A_{0} \cdot \mathrm{EUV}_{27 A}+B_{0}+\sum_{i=1}^{2}\left\{A_{i} \cdot \cos \left[\frac{2 \pi i \cdot\left(\mathrm{DoY}-\Phi_{i}\right)}{365}\right] \cdot \mathrm{EUV}_{27 A}\right. \\
& \left.+B_{i} \cdot \cos \left[\frac{2 \pi i \cdot\left(\mathrm{DoY}-\Psi_{i}\right)}{365}\right]\right\}
\end{aligned}
$$

Here, the variation slope of $\mathrm{GEC}_{27 \mathrm{~A}}$ versus $\mathrm{EUV}_{27 \mathrm{~A}}$ depends on DoY. $A_{i=1,2}\left(B_{i=1,2}\right)$ are the amplitudes of the seasonal variations of GEC versus EUV slope (intercept), and $\Phi_{\mathrm{i}=1,2}\left(\Psi_{\mathrm{i}=1,2}\right)$ correspond to the DoYs when the slope (intercept) reaches seasonal maxima. $\mathrm{A}_{0}\left(\mathrm{~B}_{0}\right)$ corresponds to the seasonal average of GEC versus EUV slope (intercept). Seasonal variations of the $\mathrm{GEC}_{27 \mathrm{~A}}$ versus $E V_{27 \mathrm{~A}}$ slope were derived via fitting $\mathrm{GEC}_{27 \mathrm{~A}}$ according to Equation 1. The correlation coefficient between the fitted $\mathrm{GEC}_{27 \mathrm{~A}}$ and the actual $\mathrm{GEC}_{27 \mathrm{~A}}$ equals to 0.994 . The black line in Fig. 7 shows the GEC $_{27 \mathrm{~A}}$ versus $\mathrm{EUV}_{27 \mathrm{~A}}$ fitting slope as a function of DoY. A prominent feature of the slope is the semiannual variation; the slope peaks at the two equinoctial seasons. The annual variation of the slope is also evident; the slope is higher at the December solstice than at the June solstice. Moreover, the slope is asymmetrical between the two equinoxes; it is higher at the March equinox than at the September equinox.

As for $G_{E C} C_{27 R}$ and $E U V_{27 R}$, Fig. $6 \mathrm{~b}$ indicates that $\mathrm{GEC}_{27 \mathrm{R}}$ nearly varies in proportion to $\mathrm{EUV}_{27 \mathrm{R}}$. $\mathrm{GEC}_{27 \mathrm{R}}$ can be estimated with $\mathrm{EUV}_{27 \mathrm{R}}$ according to the following equation when seasonal variations of the $G_{E C} C_{27 R}$ versus $E_{U V} V_{27 R}$ slope are taken into account.

$$
\mathrm{GEC}_{27 R}=\sum_{i=0}^{2}\left[a_{i} \cdot \cos \left(\frac{2 \pi i \cdot \mathrm{DoY}}{365}\right)+b_{i} \cdot \sin \left(\frac{2 \pi i \cdot \mathrm{DoY}}{365}\right)\right] \cdot \mathrm{EUV}_{27 R}
$$

Here, the proportion coefficient of $\mathrm{GEC}_{27 \mathrm{R}}$ to $\mathrm{EUV}_{27 \mathrm{R}}$ mainly includes an annual variation and a semiannual variation (similar to Equation 1). Seasonal variations of the $G_{E C} C_{27 R}$ versus $E V_{27 R}$ slope were derived via fitting $\mathrm{GEC}_{27 \mathrm{R}}$ according to Equation 2, and here the dominative 1-day lag of $\mathrm{GEC}_{27 \mathrm{R}}$ to $\mathrm{EUV}_{27 \mathrm{R}}$ was taken into account. The correlation coefficient between the fitted $\mathrm{GEC}_{27 \mathrm{R}}$ and the actual $\mathrm{GEC}_{27 \mathrm{R}}$ equals to 0.866 . The grey line in Fig. 7 shows the $\mathrm{GEC}_{27 \mathrm{R}}$ versus $\mathrm{EUV}_{27 \mathrm{R}}$ fitting

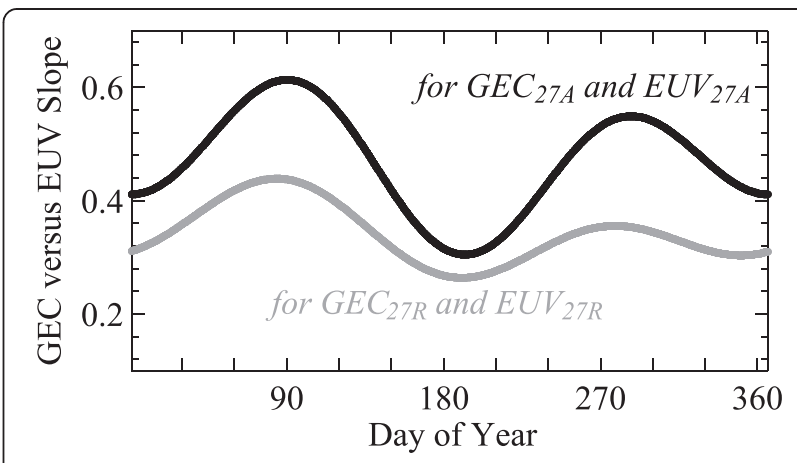

Fig. 7 Seasonal variations of GEC versus EUV fitting slopes. The black line is the fitting slope for 27-day running averaged GEC and EUV, and the grey line is the fitting slope for 27-day average residual GEC and EUV. GEC and EUV are in units of $10^{32}$ electrons and $10^{10}$ photons $/ \mathrm{cm}^{2} / \mathrm{s}$, respectively

slope as a function of DoY. The seasonal features of the $\mathrm{GEC}_{27 \mathrm{R}}$ versus $\mathrm{EUV}_{27 \mathrm{R}}$ fitting slope are similar to those of the $G_{27 \mathrm{~A}}$ versus $\mathrm{EUV}_{27 \mathrm{~A}}$ fitting slope, including the semiannual and annual variations as well as the equinoctial asymmetry. As compared with the $\mathrm{GEC}_{27 \mathrm{~A}}$ versus $E U V_{27 A}$ fitting slope, the $G_{E C} C_{27 R}$ versus $E U V_{27 R}$ fitting slope is lower in all seasons, especially at the two equinoxes. Namely, the difference between GEC responses to the solar rotation and solar cycle variations of EUV occurs in all seasons.

As compared with Fig. 7, in Fig. 6a, seasonal variations of GEC are removed; and in Fig. $6 \mathrm{~b}$, the $\mathrm{GEC}_{27 \mathrm{R}}$ versus $E_{U V} V_{27 R}$ slope is obtained by averagely fitting the data without including seasonal dependence of the slope. In fact, the GEC versus EUV fitting slopes shown in Fig. 6 are equivalent to seasonal averages of the GEC versus EUV slopes shown in Fig. 7. The average values of the GEC versus EUV slopes shown in Fig. 7 are 0.469 (in units of $10^{22}$ electrons/photon $/ \mathrm{cm}^{2} / \mathrm{s}$, for the black line) and 0.342 (for the grey line), very close to the fitting slopes shown in Fig. 6.

\begin{tabular}{|c|c|c|c|c|}
\hline \multirow[t]{2}{*}{$\begin{array}{l}\text { Latitudinal } \\
\text { interval }\end{array}$} & \multicolumn{2}{|c|}{$\begin{array}{l}\text { 365-day averaged LIEC and } \\
\text { EUV }\end{array}$} & \multicolumn{2}{|c|}{$\begin{array}{l}\text { 27-day average residual LIEC } \\
\text { and EUV }\end{array}$} \\
\hline & $\begin{array}{l}\text { Fitting } \\
\text { slope }\end{array}$ & $\begin{array}{l}\text { Correlation } \\
\text { coefficient }\end{array}$ & $\begin{array}{l}\text { Fitting } \\
\text { slope }\end{array}$ & $\begin{array}{l}\text { Correlation } \\
\text { coefficient }\end{array}$ \\
\hline $60^{\circ} \mathrm{N} \sim 90^{\circ} \mathrm{N}$ & 0.0192 & 0.996 & 0.0132 & 0.513 \\
\hline $30^{\circ} \mathrm{N} \sim 60^{\circ} \mathrm{N}$ & 0.0731 & 0.994 & 0.0535 & 0.681 \\
\hline $0^{\circ} \sim 30^{\circ} \mathrm{N}$ & 0.1710 & 0.998 & 0.1233 & 0.810 \\
\hline $0^{\circ} \sim 30^{\circ} \mathrm{S}$ & 0.1564 & 0.998 & 0.1112 & 0.819 \\
\hline $30^{\circ} \mathrm{S} \sim 60^{\circ} \mathrm{S}$ & 0.0705 & 0.997 & 0.0519 & 0.689 \\
\hline $60^{\circ} \mathrm{S} \sim 90^{\circ} \mathrm{S}$ & 0.0194 & 0.995 & 0.0136 & 0.435 \\
\hline
\end{tabular}

The variation slope of the electron density versus EUV depends on latitudes owing to the effects of ionospheric

Table 1 LIEC versus EUV fitting slopes and correlation coefficients for different latitudinal intervals 
dynamic and electrodynamics processes (e.g., Abdu et al. 2008; Chen and Liu 2010). For the global ionosphere, the difference in the variation slopes of GEC versus EUV on the two timescales was presented in the above analysis. We further investigate whether this difference occurs in different latitude regions owing to the effects of ionospheric dynamics and electrodynamics processes. Table 1 lists the variation slopes of latitudinal-integral electron content (LIEC) versus EUV and the correlation coefficients between LIEC and EUV on the two timescales for six latitudinal intervals. The LIEC versus EUV slopes and the correlation coefficients between LIEC and EUV were derived according to the method of Fig. 6. A 1-day lag was taken into account for the response of 27day average residual LIEC to EUV. The correlation between 365-day running averaged LIEC and EUV is still very high at all latitudinal intervals. For 27-day average residual LIEC and EUV, the correlation between them is higher at low latitudes but somewhat poor at high latitudes. The 27-day average residual LIEC versus EUV slope shows a feature consistent with that of the 365-day running averaged LIEC versus EUV slope, higher at low latitudes than at high latitudes. What is more important is that the difference between the solar cycle and solar rotation LIEC versus EUV slopes occurs at all latitudinal intervals. The 365-day running averaged LIEC versus EUV slope is higher than the 27-day average residual LIEC versus EUV slope by $\sim 40 \%$, consistent with the result of Fig. 6. This implies that ionospheric dynamic and electrodynamics processes, such as the fountain effect at low latitudes, are possibly not the dominative reason for the different LIEC versus EUV slopes on the two timescales.

In fact, thermospheric responses to the solar cycle and solar rotation variations of EUV are also discrepant. That has been found in previous studies. Hedin (1984) found that the fitting slope of 81-day running averaged thermospheric density versus EUV is higher than that of 81day average residual density versus EUV (see Table 3 of Hedin (1984)). It is unresolved what causes this difference in thermospheric responses to solar irradiance variations on the two timescales. The ionosphere strongly depends on the state of the thermosphere. The discrepancy between thermospheric responses to the solar cycle and solar rotation variations of EUV should result in a difference in ionospheric responses to EUV variations on the two timescales. The difference in GEC responses to EUV variations on the two timescales is consistent with that in thermospheric responses. This implies that the latter is a possible reason for the former.

\section{Conclusions}

Daily mean GEC and SOHO/SEM EUV flux were used to investigate the responses of the ionosphere to the solar cycle and solar rotation variations of EUV. The 365-day running averaged (27-day average residual) GEC and EUV were used to estimate the solar cycle (the solar rotation) variations of GEC and EUV. Two features, time lags of GEC to EUV and variation slopes of GEC versus EUV, were comparatively investigated for the solar cycle and solar rotation variations of GEC and EUV. The results indicate that GEC responses to the solar cycle and solar rotation variations of EUV are significantly different. The response of 27-day average residual GEC to EUV shows significant time lags, with EUV leading GEC. The time lag is dominated by a 1-day lag and generally presents a decrease trend with solar activity decreasing. However, there are no evident time lags for the response of 365-day running averaged GEC to EUV. The variation slope of 365-day running averaged GEC versus EUV is significantly higher than that of 27-day average residual GEC versus EUV by $\sim 40 \%$, and the difference of GEC versus EUV slope between the two timescales occurs in all seasons and at different latitudinal bands. These results present an aspect of the difference between ionospheric climatology and weather.

\section{Competing interests}

The authors declare that they have no competing interests.

\section{Authors' contributions}

YC performed the statistical analysis and drafted the manuscript. $\mathrm{LL}, \mathrm{HL}$, and $\mathrm{HZ}$ participated in the discussion of the analysis method. All authors read and approved the final manuscript.

\section{Acknowledgements}

The JPL TEC data were downloaded from this Web site ftp://cddis.gsfc.nasa.gov; the SOHO/SEM EUV data were provided by the Space Sciences Center of University of Southern California; Ap index was taken from the National Geophysical Data Center Web site. This research was supported by the National Natural Science Foundation of China (41274161, 41231065, and 41321003), Chinese Academy of Sciences (KZZD-EW-01-3), and National Important Basic Research Project of China (2012CB825604).

Received: 21 October 2014 Accepted: 18 May 2015

Published online: 27 May 2015

\section{References}

Abdu MA, Brum CGM, Batista IS, Sobral JHA, de Paula ER, Souza JR (2008) Solar flux effects on equatorial ionization anomaly and total electron content over Brazil: observational results versus IRI representations. Adv Space Res 42:617-25

Afraimovich EL, Astafyeva El, Oinats AV, Yasukevich W, Zhivetiev IV (2008) Global electron content: a new conception to track solar activity. Ann Geophys 26:335-44

Balan N, Bailey GJ, Jenkins B, Rao PB, Moffett RJ (1994) Variations of ionospheric ionization and related solar fluxes during an intense solar cycle. J Geophys Res 99:2243-53

Bilitza D (2000) The importance of EUV indices for the international reference ionosphere. Phys Chem Earth Part C 25:515-21

Bouwer SD (1992) Periodicities of solar irradiance and solar activity indices, II. Sol Phys 142:365-89

Buonsanto MJ, Pohlman LM (1998) Climatology of neutral exosphere temperature above Millstone Hill. J Geophys Res 103(A10):23381-92

Chen Y, Liu L (2010) Further study on the solar activity variation of daytime $N_{m} F_{2}$. J Geophys Res 115, A12337. doi:10.1029/2010JA015847

Chen Y, Liu L, Wan W (2011) Does the $F_{10.7}$ index correctly describe solar EUV flux during the deep solar minimum of 2007-2009? J Geophys Res 116, A04304. doi:10.1029/2010JA016301 
Chen Y, Liu L, Wan W (2012) The discrepancy in solar EUV-proxy correlations on solar cycle and solar rotation timescales and its manifestation in the ionosphere. J Geophys Res 117, A03313. doi:10.1029/2011JA017224

Chen Y, Liu L, Le H, Wan W (2014a) Geomagnetic activity effect on the global ionosphere during the 2007-2009 deep solar minimum. J Geophys Res 119:3747-54

Chen Y, Liu L, Le H, Wan W (2014b) How does ionospheric TEC vary if solar EUV irradiance continuously decreases? Earth Planets Space 66:52

Coley WR, Heelis RA (2012) Response of the equatorial topside ionosphere to 27-day variations in solar EUV input during a low solar activity period using C/NOFS. J Geophys Res 117, A03330. doi:10.1029/2011JA017301

Didkovsky L, Wieman S (2014) lonospheric total electron contents (TECS) as indicators of solar EUV changes during the last two solar minima. J Geophys Res 119:4175-84

Didkovsky LV, Judge DL, Wieman SR (2010) Minima of solar cycles 22/23 and 23/24 as seen in SOHO/CELIAS/SEM absolute solar EUV flux. In: Cranmer SR, Hoeksema JT, Kohl J (eds) SOHO-23: understanding a peculiar solar minimum. Astronomical Society of the Pacific, San Francisco

Eastes R, Bailey S, Marcos F, Wise J, Woods T (2004) The correspondence between thermospheric neutral densities and broadband measurements of the total solar soft X-ray flux. Geophys Res Lett 31, L19804. doi:10.1029/ 2004GL020801

Emmert JT, Lean JL, Picone JM (2010) Record-low thermospheric density during the 2008 solar minimum. Geophys Res Lett 37, L12102. doi:10.1029/2010GL043671

Forbes JM, Palo SE, Zhang X (2000) Variability of the ionosphere. J Atmos Sol Terr Phys 62:685-93

Hedin AE (1984) Correlations between thermospheric density and temperature, solar EUV flux, and $10.7 \mathrm{~cm}$ flux variations. J Geophys Res 89(A11):9828-34

Hocke K (2008) Oscillations of global mean TEC. J Geophys Res 113, A04302. doi:10.1029/2007JA012798

lijima BA, Harris IL, Ho CM, Lindqwister UJ, Mannucci AJ, Pi X (1999) Automated daily process for global ionospheric total electron content maps and satellite ocean altimeter ionospheric calibration based on Global Positioning System data. J Atmos Sol Terr Phys 61:1205-18

Jakowski N, Fichtelmann B, Jungstand A (1991) Solar activity control of ionospheric and thermospheric processes. J Atmos Terr Phys 53:1125-30

Judge DL, McMullin DR, Ogawa HS, Hovestadt D, Klecker B, Hilchenbach M, Möbius E, Canfield LR, Vest RE, Watts R, Tarrio C, Kühne M, Wurz P (1998) First solar EUV irradiances obtained from $\mathrm{SOHO}$ by the CELIAS/SEM. Sol Phys 177:161-73

Kane RP (2003) Fluctuations in the $27-$ day sequences in the solar index F10 during solar cycles 22-23. J Atmos Sol Terr Phys 65:1169-74

Lean J (1987) Solar ultraviolet irradiance variations: a review. J Geophys Res 92(D1):839-68

Lean J (1997) The Sun's variable radiation and its relevance for Earth. Annu Rev Astron Astrophys 35:33-67

Lean JL, Woods TN, Eparvier FG, Meier RR, Strickland DJ, Correira JT, Evans JS (2011) Solar extreme ultraviolet irradiance: present, past, and future. J Geophys Res 116, A01102. doi:10.1029/2010JA015901

Liu H, Lühr H, Henize V, Köhler W (2005) Global distribution of the thermospheric total mass density derived from CHAMP. J Geophys Res 110, A04301. doi:10.1029/2004JA010741

Liu L, Wan W, Ning B, Pirog OM, Kurkin VI (2006) Solar activity variations of the ionospheric peak electron density. J Geophys Res 111, A08304. doi:10.1029/ 2006JA011598

Liu L, Zhao B, Wan W, Ning B, Zhang M-L, He M (2009) Seasonal variations of the ionospheric electron densities retrieved from Constellation Observing System for Meteorology, lonosphere, and Climate mission radio occultation measurements. J Geophys Res 114, A02302. doi:10.1029/2008JA013819

Liu L, Chen Y, Le H, Kurkin VI, Polekh NM, Lee C-C (2011) The ionosphere under extremely prolonged low solar activity. J Geophys Res 116, A04320. doi:10.1029/2010JA016296

Mannucci AJ, Wilson BD, Yuan DN, Ho CM, Lindqwister UJ, Runge TF (1998) A global mapping technique for GPS derived ionospheric total electron content measurements. Radio Sci 33:565-82

Min K, Park J, Kim H, Kim V, Kil H, Lee J, Rentz S, Lühr H, Paxton L (2009) The 27-day modulation of the low-latitude ionosphere during a solar maximum. J Geophys Res 114, A04317. doi:10.1029/2008JA013881
Oinats AV, Ratovsky KG, Kotovich GV (2008) Influence of the 27-day solar flux variations on the ionosphere parameters measured at Irkutsk in 2003-2005. Adv Space Res 42:639-44

Pap J, Tobiska WK, Bouwer SD (1990) Periodicities of solar irradiance and solar activity indices, I. Sol Phys 129:165-89

Rich FJ, Sultan PJ, Burke WJ (2003) The 27-day variations of plasma densities and temperatures in the topside ionosphere. J Geophys Res 108(A7):1297. doi:10.1029/2002JA009731

Richards PG (2001) Seasonal and solar cycle variations of the ionospheric peak electron density: comparison of measurement and models. J Geophys Res 106:12803-19

Rishbeth H (1993) Day-to-day ionospheric variations in a period of high solar activity. J Atoms Terr Phys 55(2):165-71

Sethi NK, Goel MK, Mahajan KK (2002) Solar cycle variations of foF2 from IGY to 1990. Ann Geophys 20:1677-85

Solomon SC, Woods TN, Didkovsky LV, Emmert JT, Qian L (2010) Anomalously low solar extreme-ultraviolet irradiance and thermospheric density during solar minimum. Geophys Res Lett 37, L16103. doi:10.1029/2010GL044468

Solomon SC, Qian L, Didkovsky LV, Viereck RA, Woods TN (2011) Causes of low thermospheric density during the 2007-2009 solar minimum. J Geophys Res 116: A00H07, doi:10.1029/2011JA016508

Solomon SC, Qian L, Burns AG (2013) The anomalous ionosphere between solar cycles 23 and 24. J Geophys Res 118:6524-35

Tobiska WK, Bouwer SD (1989) Intermediate-term variations of chromospheric and coronal solar flux during high solar cycle 21 activity. Geophys Res Lett 16(8):779-82

Wang X, Eastes R, Weichecki Vergara S, Bailey S, Valladares C, Woods T (2006) On the short-term relationship between solar soft X-ray irradiances and equatorial total electron content (TEC). J Geophys Res 111: A10S15, doi:10.1029/2005JA011488

Wieman SR, Didkovsky LV, Judge DL (2014) Resolving differences in absolute irradiance measurements between the SOHO/CELIAS/SEM and the SDO/EVE. Solar Phys 289:2907-25

\section{Submit your manuscript to a SpringerOpen ${ }^{\circ}$ journal and benefit from:}

- Convenient online submission

- Rigorous peer review

- Immediate publication on acceptance

- Open access: articles freely available online

- High visibility within the field

- Retaining the copyright to your article

Submit your next manuscript at $>$ springeropen.com 